\title{
COMPARISON OF THE EFFECTIVENESS OF PLATELET-RICH PLASMA (PRP), HYALURONIC ACID AND THE COMBINATION OF BOTH IN THE TREATMENT OF MILD AND MODERATE OSTEOARTHRITIS OF THE KNEE
}

\author{
SHOMA FK ${ }^{1}$, RAHMAN MM ${ }^{2}$, CHOWDHURY ZR ${ }^{3}$, HASSAN MK ${ }^{4}$, HOSSAIN F $^{5}$, RAHMAN F ${ }^{6}$, \\ GOMES LC ${ }^{7}$, ULLAH MA ${ }^{8}$
}

\begin{abstract}
Osteoarthritis (OA) is by far the most common form of arthritis characterized by focal loss of articular cartilage, subchondral osteosclerosis, osteophyte formation at the joint margin, and remodeling of joint contour with enlargement of affected joints. This randomized, clinical trial aimed to compare the effectiveness of Platelet Rich Plasma (PRP) and Hyaluronic Acid (HA) as individual treatments and PRP in combination with $H A$ in the treatment of mild to moderate knee Osteoarthritis (OA). The study was conducted among 89 patients with mild to moderate knee osteoarthritis in the Department of Physical Medicine and Rehabilitation, Bangabandhu Sheikh Mujib Medical University (BSMMU), Dhaka, Bangladesh. Patients were randomly allocated to one of the three interventions: $H A(n=30), P R P(n=30)$, or $H A+P R P(n=29)$. Patients in HA group received 3 intra-articular knee injections with 1 week interval while patients in PRP group and $P R P+H A$ group received 2 intra-articular knee injections with 2 week interval. Functional outcome of the treatment were evaluated using the Western Ontario and McMaster Universities Arthritis Index (WOMAC) and Visual Analogue Scale (VAS) questionnaire at baseline and after 1,3,6 and 9 months of treatment. Majority of the patients in all groups were female where mean age of the patients were about 50 years. Pain scores in VAS scale significantly reduced in PRP group when compared to HA group and also in PRP+HA when compared to HA group which persist till nine months $(p<0.05)$. No significant statistical difference was found in VAS scores between PRP and $P R P+H A$ group. WOMAC pain scores also significantly reduced in PRP group when compared to $H A$ group and also in PRP+HA when compared to HA group $(p<0.05)$. WOMAC stiffness significantly reduced in $P R P+H A$ group when compared to HA group at three month $(p<0.001)$ and at six month $(p=0.011)$. At nine month, physical activity scores significantly reduced in $P R P$ group compared to HA group $(p=0.002)$ and in PRP+HA when compared to HA group $(p<0.001)$. No significant reduction was observed in WOMAC scores when the outcome of PRP+HA was compared with PRP alone. PRP provides better functional outcome than HA. The combination of $P R P$ and HA also provides better outcome than HA alone but does not provide better outcome than PRP alone.
\end{abstract}

Key words: Platelet-Rich Plasma (PRP), Hyaluronic Acid, Osteoarthritis of the knee.

J Dhaka Med Coll. 2019; 28(2) : 172-178

1. Dr. Farzana Khan Shoma, Assistant Professor, Department of Physical Medicine and Rehabilitation, Bangabandhu Sheikh Mujib Medical University, Dhaka

2. Md. Mahmudur Rahman, Associate Professor, Department of Physical Medicine and Rehabilitation, Bangabandhu Sheikh Mujib Medical University, Dhaka, Bangladesh.

3. Ziaur Rahman Chowdhury, Research Assistant, Department of Physical Medicine and Rehabilitation, Bangabandhu Sheikh Mujib Medical University, Dhaka, Bangladesh.

4. Dr. Muhammad Kamrul Hassan, Consultant, Department of Physical Medicine and Rehabilitation, Retaired government employee hospital, Dhaka, Bangladesh.

5. Farzana Hossain MPH. Freelance researcher, Kakrail, Dhaka, Bangladesh.

6. Dr. Farzana Rahman FCPS. Assistant Professor, Department of Hematology, Bangabandhu Sheikh Mujib Medical University, Dhaka, Bangladesh.

7. Dr. Lilian Catherene Gomes, Medical Officer, Department of Physical Medicine and Rehabilitation, Bangabandhu Sheikh Mujib Medical University, Dhaka, Bangladesh.

8. Prof. Md. Ahsan Ullah, Professor, Department of Physical Medicine and Rehabilitation, Bangabandhu Sheikh Mujib Medical University, Dhaka, Bangladesh.

Correspondence: Dr. Farzana Khan Shoma, Assistant Professor, Department of Physical Medicine and Rehabilitation, Bangabandhu Sheikh Mujib Medical University, Dhaka, Bangladesh, Contact No. 01777400163, E-mail: farzanadmck 53@gmail.com

Received: 05-05-2020

Revision: 14-07-2020

Accepted: 11-08-2020 


\section{Introduction}

Osteoarthritis (OA) is by far the most common form of arthritis characterized by focal loss of articular cartilage, subchondral osteosclerosis, osteophyte formation at the joint margin, and remodeling of joint contour with enlargement of affected joints. ${ }^{1}$ It is strongly associated with age. Over $80 \%$ of people over 55 years of age have OA at least one joint. Knee OA is one of the most important because of high prevalence of pain and disability that they cause in older adults and the massive health care resource input that results from this. ${ }^{2}$

The targets of OA treatment are pain reduction, func-tion and mobility improvement, prevention or correction of the deformity, and slowing the progression of the disease. There are numerous conservative treatments for knee OA with benefits and disadvan-tages. ${ }^{3}$ For example, Non-steroidal anti-inflammatory drugs (NSAIDs) and intra-articular corticosteroid are common treatments of arthritis. Despite their low cost and easy access, these treatments have systemic adverse effects and may cause joint cartilage destruction and flare up of the osteoarthritic pro-cess. ${ }^{4}$

Hyaluronic Acid (HA) is a naturally occurring glycosaminoglycan and a component of Synovial Fluid and cartilage matrix. Synovial cells, fibroblasts and chondrocytes synthesize Hyaluronic Acid and secrete into the joint. Hyaluronic Acid enhances viscosity and elastic nature of Synovial Fluid. ${ }^{5}$ The efficacy of intraarticular HA injection for the treatment of OA knees remains a matter of conflict. A Cochrane review $^{6}$ and recent systematic review and metaanalysis ${ }^{7}$ concluded that HA had beneficial effects on pain, functional improvement. However, several meta-analyses contrarily reported that intra-articular HA injections were not clinically effective and might even be associated with a greater risk of adverse effects. ${ }^{8,9,10}$

PRP is an autologous concentration of human platelets which contains growth factors, cytokines, and many other mediators. ${ }^{11,} 12$ It has antinociceptive and anti-inflammatory activities to reduce pain and modulate the OA process. ${ }^{13} \mathrm{PRP}$ with its potent mixture of growth factors and cytokines has also been shown to increase the production of HA from native synoviocytes. ${ }^{14}$ Thus, it is hypothesized that their combination may be synergistic. According to literature, combining PRP and HA may benefit from their dissimilar biological mechanisms and helping with the signaling molecules as inflammatory molecules, catabolic enzymes, cytokines and growth factors. Also, it was demonstrated that the association of HA+PRP showed synergic effects in the potentials regenerative and anti-inflammatory in comparison to HA or PRP alone. This association can alter the inflammatory cytokines in the degeneration process of the chondrocytes through specific mediators (CD44, TGF-âRII) and also promote the regeneration of cartilage and inhibit inflammation in OA. ${ }^{15,16}$

However, there is not much research examining such synergistic effects in human. Hence the present study aimed to compare the effectiveness of Platelet Rich Plasma (PRP) and Hyaluronic Acid (HA) as individual treatments and PRP in combination with $\mathrm{HA}$ in the treatment of mild to moderate knee Osteoarthritis (OA).

\section{Methods}

Research design: The study was a randomized, clinical trial, conducted among 89 patients with mild to moderate knee osteoarthritis in the Department of Physical Medicine and Rehabilitation, Bangabandhu Sheikh Mujib Medical University (BSMMU), Dhaka, Bangladesh. Patients with primary osteoarthritis of the target knee fulfilling the ACR clinical criteria were selected for the study. Patients having history/ evidence of acute swollen joint (septic arthritis), recent trauma, fracture, unstable knee joint, Tubercular arthritis, Crystalline arthritis, Inflammatory disease (e.g. rheumatoid arthritis, psoriatic arthritis), peripheral neuropathy/Neurological deficit of lower extremities were excluded from the study. Patient who had received intraarticular corticosteroid injection or PRP injection over the previous 6 months or viscosupplementation to the target knee and underwent joint replacement or arthoplasty on the target knee or any surgical procedure 
scheduled in the next six months were also excluded from the study. Patients were randomly allocated by lottery to one of the three interventions: HA $(n=30)$, PRP $(n=30)$, or HA+PRP $(n=29)$.

Blood sample collection and PRP preparation: Selected patients were sent to transfusion medicine department. There about $30 \mathrm{ml}$ blood was collected to prepare platelet rich plasma. Blood was collected in sterile procedure (venepuncture techniuue almost identical to having a standard blood collection for pathology testing) and allowed it to spin in a centrifuge machine for 15 minutes about 3200 $\mathrm{rpm}$. Then blood was separated into its various constituents; red blood cells on the bottom, plasma on the top, and platelets in the middle. On the top of the red blood cell layer, there was buffy coat. Then buffy coat was extracted which was the Platelet rich plasma. This platelet rich plasma was used in our study. The entire process took less than 30 minutes.

Details of Treatment: With aseptic technique, injection was administered through a direct parapatellar approach. Preadministration of anesthetic skin spray or subcutaneous local anesthetics was permitted.

Patients in HA group received 3 intra-articular knee injections $(4 \mathrm{ml})$ with 1 week interval.

Patients in PRP group received 2 intra-articular knee injections $(10 \mathrm{ml})$ with 2 week interval.

Patients in PRP+HA group received 2 intraarticular knee injections $(6 \mathrm{ml} \mathrm{PRP}$ and $4 \mathrm{ml}$ HA) with 2 week interval.

The patient was then observed for 15-20 min and then discharged.

Post-injection protocol: The use of NSAID was prohibited. Because there might be discomfort experienced by the patient at the site of the injection for up to 48 hours, patients were encouraged to ice the injection site, elevate the limb and modify activities. Patients were discharged to home with instruction to limit their activities for 48 hours.

Respondents of both groups were assessed to see the effects of treatment at $4^{\text {th }}$ week, $12^{\text {th }}$ week, $24^{\text {th }}$ week and $36^{\text {th }}$ week.
Paracetamol was allowed for break-thru pain < 2000mg/day.

Quadriceps strengthening exercise was advised in the form of extension of knees 10 repetition 2 times daily.

Instruction for activity of daily living (ADLs) was prescribed for all patients.

Outcome Measures: Outcome were measured by OA specific translated and validated Bengali instrument- Western Ontario and McMaster Universities Osteoarthritis Index (WOMAC) questionnaire: WOMAC is a 24-item self-report questionnaire, that include Pain score (5 domain), Stiffness score (2 domain), Physical function score (17 domain). All scores were converted into a scale of 0 to 100 for better representation.

Pain score was collected on visual analogue scale (VAS 0-10).

Data processing and analysis: The statistical analysis was conducted using SPSS (statistical package for social science) version 25 statistical software. Descriptive analyses are provided for demographic and clinical characteristics. Independent sample $t$ test was applied to compare the continuous data. Here, $p<0.05$ was considered significant. Here, all $\mathrm{p}$-values were two sided.

Ethical implication: Ethical clearance was taken from Institutional Review Board (IRB) of BSMMU. Informed written consent was taken from every patient before enrollment.

\section{Results}

Demographic and clinical characteristics: Majority of the patients in all groups were female where mean age of the patients were about 50 years. Majority of patients have grade III of OA by Kellgren-Lawrence in the left knee (table 1).

VAS scores: At baseline there was no significant difference between the groups regarding pain on VAS scale. Significant statistical difference was observed in VAS scores from one month in PRP vs HA group and HA vs PRP+ HA group which persist till nine months $(p<0.05)$. No significant difference was found in VAS scores in PRP vs PRP+ HA group (table 2). 
WOMAC scores: Significant statistical difference was observed in WOMAC pain scores from one month in PRP vs HA group and HA vs $\mathrm{PRP}+\mathrm{HA}$ group which persist till nine months $(p<0.05)$. No significant difference was found in pain scores in PRP vs PRP+ HA group. At nine month, physical activity scores significantly reduced in PRP vs HA group and HA vs PRP+ HA group. No significant reduction was observed in WOMAC scores when the outcome of PRP+HA was compared with PRP alone (table 3).

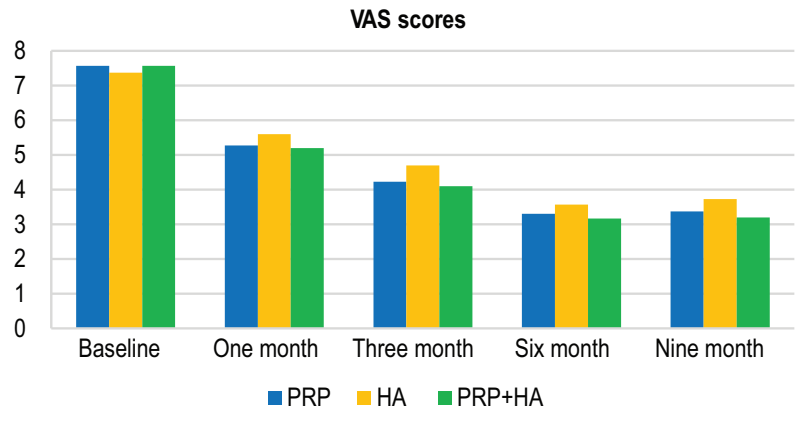

Fig.-1: VAS scores of the patients

Table-I

Demographic characteristic of the patients

\begin{tabular}{lccc}
\hline Criteria & PRP group & HA group & PRP + HA group \\
\hline Gender & $12(40.0)$ & $13(43.3)$ & $14(48.3)$ \\
Male & $18(60.0)$ & $17(56.7)$ & $15(51.7)$ \\
Female & $49.27 \pm 6.82$ & $52.63 \pm 5.40$ & $51.57 \pm 4.85$ \\
Age (mean \pm SD) & $14.63 \pm 6.62$ & $16.67 \pm 6.37$ & $14.87 \pm 5.46$ \\
Disease duration (months) (mean \pm SD) & & & \\
Kellgren-Laurence grade & $14(46.7)$ & $16(52.2)$ & $9(31.1)$ \\
2 & $16(52.2)$ & $14(46.7)$ & $20(68.9)$ \\
3 & & & \\
Side involvement & $15(50.0)$ & $14(46.7)$ & $12(41.4)$ \\
Right & $15(50.0)$ & $16(52.2)$ & $17(58.6)$ \\
Left & & & \\
\hline
\end{tabular}

Table-II

Comparison of VAS scores of the patients

\begin{tabular}{lcccccc}
\hline VAS scores & PRP & HA & PRP + HA & \multicolumn{3}{c}{ p values } \\
\cline { 5 - 7 } & group & group & group & PRP vs & PRP vs & HA vs \\
& & & & HA & PRP+ HA & PRP+ HA \\
\hline At baseline & $7.57 \pm 0.57$ & $7.37 \pm 0.61$ & $7.57 \pm 0.50$ & 0.196 & 1.000 & 0.174 \\
At one month & $5.27 \pm 0.45$ & $5.60 \pm 0.49$ & $5.20 \pm 0.76$ & 0.009 & 0.681 & 0.019 \\
At three month & $4.23 \pm 0.43$ & $4.70 \pm 0.47$ & $4.10 \pm 0.30$ & $<0.001$ & 0.172 & $<0.001$ \\
At six month & $3.30 \pm 0.47$ & $3.57 \pm 0.50$ & $3.17 \pm 0.38$ & 0.038 & 0.229 & 0.001 \\
At nine month & $3.37 \pm 0.49$ & $3.73 \pm 0.45$ & $3.20 \pm 0.41$ & 0.004 & 0.157 & $<0.001$ \\
\hline
\end{tabular}


Table-III

Comparison of WOMAC pain, WOMAC stiffness and WOMAC physical activity scores of the patients

\begin{tabular}{lcccccc}
\hline Criteria & $\begin{array}{c}\text { PRP } \\
\text { group }\end{array}$ & $\begin{array}{c}\text { HA } \\
\text { group }\end{array}$ & $\begin{array}{c}\text { PRP }+ \text { HA } \\
\text { group }\end{array}$ & $\begin{array}{c}\text { PRP values } \\
\text { HA }\end{array}$ & $\begin{array}{c}\text { PRP vs } \\
\text { PRP+ HA }\end{array}$ & $\begin{array}{c}\text { HA vs } \\
\text { PRP HA }\end{array}$ \\
\hline At base line & & & & & & \\
Pain & $375.23 \pm 5.34$ & $377.50 \pm 4.10$ & $374.83 \pm 4.82$ & 0.070 & 0.762 & 0.025 \\
Stiffness & $154.50 \pm 6.99$ & $153.50 \pm 8.72$ & $157.33 \pm 8.17$ & 0.626 & 0.154 & 0.084 \\
Physical activity & $1435.00 \pm 78.95$ & $1445.00 \pm 86.45$ & $1471.33 \pm 70.44$ & 0.642 & 0.065 & 0.201 \\
At one month & & & & & & \\
Pain & $362.00 \pm 5.51$ & $365.17 \pm 4.45$ & $360.50 \pm 6.34$ & 0.017 & 0.332 & 0.002 \\
Stiffness & $126.00 \pm 12.06$ & $128.00 \pm 10.22$ & $129.50 \pm 15.10$ & 0.491 & 0.325 & 0.654 \\
Physical activity & $1340.00 \pm 74.74$ & $1364.33 \pm 76.84$ & $1336.67 \pm 92.78$ & 0.219 & 0.879 & 0.213 \\
At three month & & & & & & \\
Pain & $349.67 \pm 3.92$ & $354.17 \pm 3.96$ & $346.83 \pm 8.76$ & $<0.001$ & 0.101 & $<0.001$ \\
Stiffness & $117.33 \pm 12.29$ & $122.00 \pm 4.67$ & $112.67 \pm 10.96$ & 0.057 & 0.126 & $<0.001$ \\
Physical activity & $1235.33 \pm 79.73$ & $1257.00 \pm 67.93$ & $1221.67 \pm 117.21$ & 0.262 & 0.600 & 0.159 \\
At six month & & & & & & \\
Pain & $330.17 \pm 10.29$ & $336.00 \pm 7.12$ & $326.50 \pm 8.72$ & 0.013 & 0.142 & $<0.001$ \\
Stiffness & $96.67 \pm 10.19$ & $98.00 \pm 5.66$ & $92.33 \pm 10.23$ & 0.534 & 0.106 & 0.011 \\
Physical activity & $1110.00 \pm 71.39$ & $1138.00 \pm 68.80$ & $1108.33 \pm 128.04$ & 0.127 & 0.951 & 0.270 \\
At nine month & & & & & & \\
Pain & $331.83 \pm 10.29$ & $339.83 \pm 7.93$ & $328.17 \pm 6.76$ & $<0.001$ & 0.075 & $<0.001$ \\
Stiffness & $98.83 \pm 10.19$ & $97.00 \pm 3.11$ & $93.33 \pm 9.41$ & 0.122 & 0.484 & 0.050 \\
Physical activity & $1058.00 \pm 67.64$ & $1117.33 \pm 76.83$ & $1025.00 \pm 76.28$ & 0.002 & 0.081 & $<0.001$ \\
\hline
\end{tabular}

\section{Discussion}

Osteoarthritis is a degenerative joint disease that occurs primarily in older individuals, characterized by erosion of the articular cartilage, hypertrophy of bone at the margins (i.e., osteophytes), subchondral sclerosis, and a range of biochemical and morphologic alterations of the synovial membrane and joint capsule. In early stage, anabolic changes, characterized by proliferation of chondrocytes and increased matrix production, are followed by a predominantly catabolic state, characterized by decreased matrix synthesis, increased proteolytic degradation of matrix, and chondrocyte apoptosis. Many of the features of the chondrocyte in the catabolic state are related to the production of inflammatory mediators by synovium and chondrocytes that act locally to perpetuate cartilage degradation. ${ }^{17}$

Pain is the man clinical problem of OA knee. After intervention, VAS scores significantly decreased in PRP group compared to HA group and PRP+HA group. HA when added to PRP did not provide any extra effect on pain reduction. Other studies also found superior result when treated with PRP compared to HA. ${ }^{18,} 19,20$ The platelet concentrate is activated by addition of calcium chloride, which results in the formation of platelet gel and this stimulate the release of growth factors and bioactive molecules. ${ }^{21}$ Therefore, platelets actively PRP actively participate in healing processes by delivering a broad spectrum of growth factors (insulin.like growth factor, transforming growth factor b.I, platelet.derived growth factor, and many others) and other active molecules (e.g., arachidonic acid metabolites, cytokines, chemokines, ascorbic acid, extracellular matrix proteins, and nucleotides) to the injured site. ${ }^{22}$ These factors altogether contribute to comprehensive roles of PRP, including anti.inflammation, angiogenesis, chondrogenesis, chondrocyte proliferation, bone remodeling, coagulation, and cell differentiation and this, in turn, reduces inflammation and pain. ${ }^{23}$ 
Response rate was quicker in VAS scale than WOMAC scale. When clinical outcome was measured in WOMAC scale, only pain significantly decreased in PRP group compared to HA group. Pain also significantly decreased in PRP+HA group compared to HA group. However, other subscales like stiffness and physical activity showed no difference between groups till six months. At nine month of treatment, physical activity improved significantly in PRP group compared to HA group which was also prominent in PRP+HA group compared to HA group. This supports the findings of other studies that showed PRP having superior results versus HA in the treatment of knee OA. Sánchez et al. showed that PRP is better in pain, physical activity and overall WOMAC scores in 5 weeks compared to HA. ${ }^{24}$ Spaková et al. showed statistically significant better results in the PRP group compared to HA at 3 and 6 month follow up periods in WOMAC scores. ${ }^{25}$ Kon et al. showed that the PRP group showed better results than the HA group at 6 months follow up in the International Knee Documentation Committee and VAS scores and concluded that autologous PRP injections showed more and longer efficacy than HA injections in reducing pain and symptoms and recovering articular function. ${ }^{20}$ Raeissadat et al. had conducted a non-placebocontrolled randomized clinical trial among 160 patients affected by knee OA. In the PRP group, two intra-articular injections at 4-week interval were applied, and in the HA group, three doses of intra-articular injection at 1-week interval were applied. At the 12-month follow-up, WOMAC pain score and bodily pain significantly improved in both groups; however, better results were deter-mined in the PRP group compared to the HA group $(\mathrm{p}<0.001) .{ }^{18}$

PRP was found superior in terms of pain reduction and functional improvement in knee when compared to HA. However, the combination of PRP+HA did not prove superior to PRP though it was found superior to HA. Separately HA and PRP are beneficial for joint cells although they function through different mechanisms. Anitua et al. evaluated the potential of pure PRP to induce tendon cells and synovial fibroblasts migration and examined whether the combination of PRP with HA improves their motility in vitro. PRP stimulated the migration of fibroblasts, as well as HA, but this effect was more prominent when HA was combined with PRP. Indeed, an increase of $335 \%$ in motility was observed in the case of HA+PRP treatment compared with HA. Therefore, this 'in vitro' study definitely proves that PRP improves the biological properties of HA. ${ }^{26}$ The randomized controlled trial which evaluated the effectiveness of HA and PRP as monotherapies for mild to moderate $\mathrm{OA}$ and compare the results to the combination of $\mathrm{PRP}+\mathrm{HA}$, found that the combination of $\mathrm{HA}$ and $\mathrm{PRP}$ resulted to better outcomes than HA alone up to 1 year and PRP alone up to 3 months. ${ }^{27}$

\section{Conclusion}

PRP provides better functional outcome than HA. The combination of PRP and HA also provides better outcome than HA alone but does not provide better outcome than PRP alone.

\section{Acknowledgments}

Transfusion medicine department of BSMMU for technical support.

\section{Conflict of Interest: None}

\section{References}

1. Clunie GPR, Ralston SH. Rheumatology and bone disease. In: Ralston SH, Penman ID, Strachan MW, Hobson RP, editors. Davidson's Principles and Practice of Medicine. 23 $3^{\text {rd }}$ edition. Philadelphia: Elsevier Saunders; 2018. pp. 981-1060.

2. Dieppe P, Blom, A. Osteoarthritis. In: Blom A, Warwick D, Whitehouse, M editors. Apley \& Solomon's System of Orthopaedics and Trauma. $10^{\text {th }}$ edition. Boca Raton: CRC Press; 2018. pp. 91-105.

3. McArthur BA, Dy CJ, Fabricant PD, Gonzalez DVA. Long term safety, effi-cacy, and patient acceptability of hyaluronic acid injection in patients with pain-ful osteoarthritis of the knee. Patient Prefer Adherence. 2012;6:905-10.

4. Kon E, Filardo G, Drobnic M, Madry H, Jelic M, van Dijk N, Della Villa S. Non-surgical management of early knee osteoarthritis. Knee Surgery, Sports Traumatology, Arthroscopy. 2012;20(3):436-49.

5. Ayhan E, Kesmezacar H, Akgun I. Intraarticular injections (corticosteroid, hyaluronic acid, platelet rich plasma) for the knee osteoarthritis. World journal of orthopedics. 2014;5(3):351..

6. Bellamy N, Campbell J, Welch V, Gee TL, Bourne R, Wells GA. Viscosupplementation for the treatment of osteoarthritis of the knee. Cochrane database of systematic reviews. 2006(2). 
7. Miller LE, Block JE. US-approved intra-articular hyaluronic acid injections are safe and effective in patients with knee osteoarthritis: Systematic review and metaanalysis of randomized, saline-controlled trials. Clinical Medicine Insights: Arthritis and Musculoskeletal Disorders.2013;6:57-63.

8. Arrich J, Piribauer F, Mad P, Schmid D, Klaushofer $\mathrm{K}$, Müllner M. Intra-articular hyaluronic acid for the treatment of osteoarthritis of the knee: systematic review and meta-analysis. Canadian Medical Association Journal. 2005;172(8):1039-43.

9. Reichenbach S, Blank S, Rutjes AW, Shang A, King EA, Dieppe PA, Jüni P, Trelle S. Hylan versus hyaluronic acid for osteoarthritis of the knee: A systematic review and meta analysis. Arthritis care \& research. 2007;57(8):1410-8.

10. Filardo G, Kon E, Di Martino A, Di Matteo B, Merli ML, Cenacchi A, Fornasari PM, Marcacci M. Plateletrich plasma vs hyaluronic acid to treat knee degenerative pathology: study design and preliminary results of a randomized controlled trial. BMC musculoskeletal disorders. 2012;13(1):229.

11. Foster TE, Puskas BL, Mandelbaum BR, Gerhardt MB, Rodeo SA. Platelet-rich plasma: from basic science to clinical applications. The American journal of sports medicine. 2009;37(11):2259-72.

12. Senet P, Bon FX, Benbunan M, Bussel A, Traineau R, Calvo F, Dubertret L, Dosquet C. Randomized trial and local biological effect of autologous platelets used as adjuvant therapy for chronic venous leg ulcers. Journal of vascular surgery. 2003;38(6):1342-8.

13. Sundman EA, Cole BJ, Karas V, Della Valle C, Tetreault MW, Mohammed HO, Fortier LA. The antiinflammatory and matrix restorative mechanisms of platelet-rich plasma in osteoarthritis. The American journal of sports medicine. 2014;42(1):35-41.

14. Anitua E, Sanchez M, Nurden AT, Zalduendo MM, De La Fuente M, Azofra J, Andia I. Platelet-released growth factors enhance the secretion of hyaluronic acid and induce hepatocyte growth factor production by synovial fibroblasts from arthritic patients. Rheumatology. 2007;46(12):1769-72.

15. Andia I, Abate M. Knee osteoarthritis: hyaluronic acid, platelet-rich plasma or both in association? Expert Opinion on Biological Therapy. 2014;14(5):635-49.

16. Chen W, Lo W, Hsu W, Wei H, Liu H, Lee C, Chen ST, Shieh Y, Williams DF, Deng W. Synergistic anabolic actions of hyaluronic acid and platelet-rich plasma on cartilage regeneration in osteoarthritis therapy. Biomaterials. 2014;35(36):9599-607.

17. Di Cesare PE, Haudenschild DR, Samuels J, Abramson SB. Pathogenesis of osteoarthritis. In: Firestein GS, Gabriel SE, Mcinnes IB, O'Dell JR, editors. Kelly's Textbook of Rheumatology. $10^{\text {th }}$ edition. Philadelphia: Elsevier Saunders; 2017.p.1685.

18. Raeissadat SA, Rayegani SM, Hassanabadi H, Fathi M, Ghorbani E, Babaee M, Azma K. Knee osteoarthritis injection choices: hyaluronic acid versus platelet rich plasma. Clinical Medicine Insights: Arthritis and Musculoskeletal Disorders. 2015;7(8):1-8.

19. Chang KV, Hung CY, Aliwarga F, Wang TG, Han DS, Chen WS. Comparative effectiveness of platelet-rich plasma injections for treating knee joint cartilage degenerative pathology: a systematic review and meta-analysis. Archives of physical medicine and rehabilitation. 2014 Mar 1;95(3):562-75.

20. Kon E, Mandelbaum B, Buda R, Filardo G, Delcogliano M, Timoncini A, Fornasari PM, Giannini S, Marcacci M. Platelet-rich plasma intra-articular injection versus hyaluronic acid viscosupplementation as treatments for cartilage pathology: from early degeneration to osteoarthritis. Arthroscopy: The Journal of Arthroscopic \& Related Surgery. 2011;27(11):1490-501.

21. Anitua E, Andia I, Ardanza B, Nurden P, Nurden AT. Autologous platelets as a source of proteins for healing and tissue regeneration. Thrombosis and haemostasis. 2004;91(01):4-15.

22. Pietrzak WS, Eppley BL. Platelet rich plasma: biology and new technology. Journal of Craniofacial Surgery. 2005 Nov 1;16(6):1043-54.

23. Drengk A, Zapf A, Stürmer EK, Stürmer KM, Frosch $\mathrm{KH}$. Influence of platelet rich plasma on chondrogenic differentiation and proliferation of chondrocytes and mesenchymal stem cells. Cells Tissues Organs 2009; 189:317 26.

24. Sánchez M, Anitua E, Azofra J, Aguirre JJ, Andia I. Intra-articular injection of an autologous preparation rich in growth factors for the treatment of knee OA: a retrospective cohort study. Clinical and Experimental Rheumatology. 2008;26(5):910-3.

25. Spaková T, Rosocha J, Lacko M, Harvanová D, Gharaibeh A. Treatment of knee joint osteoarthritis with autologous platelet-rich plasma in comparison with hyaluronic acid. American Journal of Physical Medicine \& Rehabilitation. 2012;91(5):411-7.

26. Anitua E, Sanchez M, De la Fuente M, Zalduendo $\mathrm{MM}$, Orive G. Plasma rich in growth factors (PRGFEndoret) stimulates tendon and synovial fibroblasts migration and improves the biological properties of hyaluronic acid. Knee Surgery, Sports Traumatology, Arthroscopy. 2012;20(9):1657-65.

27. Lana JF, Weglein A, Sampson SE, Vicente EF, Huber SC, Souza CV, Ambach MA, Vincent H, Urban-Paffaro A, Onodera CM, Annichino-Bizzacchi JM. Randomized controlled trial comparing hyaluronic acid, platelet-rich plasma and the combination of both in the treatment of mild and moderate osteoarthritis of the knee. Journal of stem cells \& regenerative medicine. 2016;12(2):69. 